\title{
Binding affinity between small molecules in solvent and polymer film using molecular dynamics simulations
}

\author{
Enqiang Lin ${ }^{\mathrm{a}}$, Xiaorong You ${ }^{\mathrm{b}}$, Robert M. Kriegel ${ }^{\mathrm{b}}$, Ronald D. Moffitt ${ }^{\mathrm{c}}$, Romesh C. Batra ${ }^{\mathrm{a}, *}$ \\ a Department of Biomedical Engineering and Mechanics, M/C 0219, Virginia Polytechnic Institute and State University, Blacksburg, VA 24061, USA \\ b Global Research, The Coca-Cola Company, 1 Coca-Cola Plaza, Atlanta, GA 30313, USA \\ ' Global Engineering, The Coca-Cola Company, 1 Coca-Cola Plaza, Atlanta, GA 30313, USA
}

\section{H I G H L I G H T S}

- A novel free energy method is proposed to assess the binding affinity of small molecules to the polymeric film.

- Binding affinity of aldehydes (C8, C9, C10), Eugenol and D-limonene to a polymer film is studied.

- The aldehydes have much higher affinity than the Eugenol and the Dlimonene.

- The small molecules exhibit increased affinity to the polymer film as the temperature increases.

\section{A R T I C L E I N F O}

\section{Article history:}

Received 14 October 2016

Received in revised form 8 February 2017

Accepted 24 February 2017

Available online 27 February 2017

\section{Keywords:}

Binding affinity

Polymeric film

Adsorption

Molecular simulation

Metadynamics
G R A P H I C A L A B S T R A C T

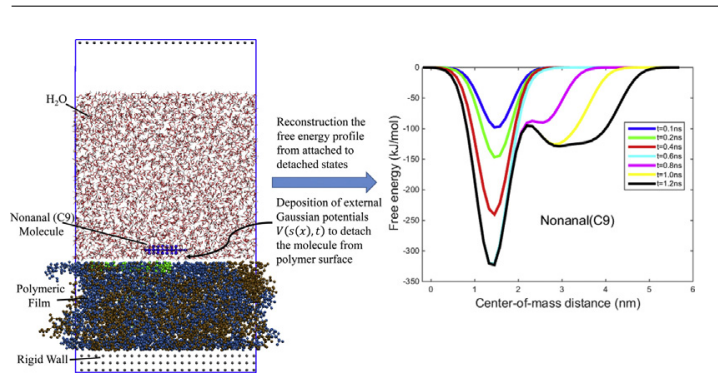


imbalances involve both the process of adsorption and diffusion [1], i.e., the adsorption of small molecules onto the polymer surface followed by their dissolution and diffusion into the polymer interior under the driving force of concentration gradient. Though the technological significance of the small molecule-polymer interaction has driven a large number of experimental [2-4] and theoretical studies [5-7] during the last decades, only a few studies have focused on understanding fundamentals of the adsorption process that is useful not only in polymeric barrier material design and selection but also in quantitative identification of important factors that govern the small molecule adsorption.

The small molecules adsorption depends upon the relative attraction forces or mutual binding affinities between the molecules and the polymeric films. These binding affinities are governed by thermodynamics of the interaction system and can be characterized by the solubility parameter $\left(\delta, \mathrm{J}^{1 / 2} \mathrm{~m}^{-3 / 2}\right)$ on the basis of Hildebrand's theory. Experimental methods (e.g., the gravimetric technique [8,9], inverse gas chromatography (IGC) $[10,11]$ and Fourier transform infrared (FT-IR) spectroscopy $[4,12])$, and theoretical analyses (e.g., group contribution methods [5-7]) have been used to estimate the solubility parameter and the small moleculepolymer binding affinity. These experimental methods are mainly based on the sorption kinetic determination under either static or dynamic conditions and are often used to compare the barrier property of different polymer films to several small molecules. However, they are expensive, time consuming and labor intensive. Depending upon the experimental method used, large variations in the measured solubility are found in the literature [3]. The theoretical methods determine the solubility through the analysis of structural fragments within the molecule [5-7], and are typically applicable for systems with simple molecular structures and dominant van der Waals forces. They usually cannot deal with complex molecules with unknown structures or those containing hydrogen and longrange interactions. Therefore, one needs alternative methods to evaluate the affinity and miscibility of small molecules in polymeric matrix for understanding their interactions and the phenomenon of concentration imbalances.

Molecular dynamics (MD) simulations have been shown to be a powerful method to describe interactions among materials as they can provide structural and dynamics details that are difficult to assess in experiments. With an appropriate interaction potential, MD simulations can predict many thermodynamics and transport properties of small molecules in a polymeric film such as the solubility parameter [13-15], the partition coefficient [16,17] and the diffusion coefficient [18-20]. In MD simulations, the prediction of binding affinity between two components is characterized through the solubility parameter $\delta$. The role of $\delta$ can be understood from the concept of cohesive energy density $E_{\mathrm{CED}}$, the enthalpy change of mixing $\Delta H_{m}$ and the corresponding Gibbs free energy of mixing $\Delta G_{m}$, which are defined as $[21,22]$

$$
\begin{aligned}
& \delta=\left(E_{\mathrm{CED}}\right)^{1 / 2}=\left[\frac{\Delta E_{v}}{V_{m}}\right]^{1 / 2}=\left[\frac{\Delta H_{v}-R T}{V_{m}}\right]^{1 / 2} \\
& \Delta H_{m}=V\left(\delta_{1}-\delta_{2}\right)^{2} \phi_{1} \phi_{2} \\
& \Delta G_{m}=\Delta H_{m}-T \Delta S_{m}
\end{aligned}
$$

Here $V_{m}$ is component's molar volume, $\Delta E_{v}$ the molar energy of vaporization, $\Delta H_{v}$ the molar enthalpy of vaporization, $R$ the universal gas constant, $T$ the absolute temperature, $V$ the volume of the mixture of two components, $\Delta S_{m}$ the entropy of mixing, and $\phi_{1}, \phi_{2}$ are volume fractions of components 1 and 2, respectively. For binding or mixing to occur (i.e., $\Delta G_{m}<0$ ), it is necessary that the solubility parameters of the two components should be close to each other, since $T \Delta S_{m}$ is usually positive. Though the binding affinity using $\delta$ can be characterized for small molecule-polymer systems, it has two main drawbacks. First, the determination of $\delta$ for small molecules and a polymeric film requires calculation of the non-bonded cohesive energy in their gas phases (Eq. (1)). For a complex polymeric film with long chains and cross-linked structures, this calculation is problematic as conformations of the polymer in the gas phase are quite different from those in the condensed state. Second, this method cannot deal with the more realistic conditions when small molecule-polymer binding occurs in an aqueous solution. The presence of water makes it difficult to determine the exact cohesive energy between small molecules and the polymer.

Here we present an alternative method to overcome the drawbacks of the solubility parameter-based method for determining the binding affinity of small molecules in a polymeric film. The method is based on calculation of the adsorption free energy (Eq. (3)) that is determined from the reversible thermodynamics work by detaching adsorbed small molecules from the polymer surface. The difficulty with this technique is the large number of degrees of freedom of the binary system and their corresponding high energy barriers between different conformations. Enhanced sampling methods [23-26] must be used to speed up and sample all conformational configurations that are unreachable using the standard MD simulations. In this work, the Metadynamics method [24] that uses a biased potential to drive a simulation towards certain reaction coordinates is used to enhance conformational sampling. The developed simulation method is a useful tool to obtain a clear picture of the molecular level mechanisms of a small molecule's adsorption in the solution and on a polymer surface.

The objective of this work is to use the free energy-based binding affinity assessment method to ascertain the binding affinity of small molecules to a polymeric film that plays a critical role in the process of concentration loss. A commercially available polymeric film that consists of four different constituents is simulated and tested with five small molecules (C8, C9, C10, Eugenol and D-limonene) to assess their mutual binding affinities in an aqueous solution. The presence of four compositions to form a complex microstructure in this polymeric film makes it very difficult to apply the solubility parameter-based method for the binding affinity analysis.

The outline of this paper is as follows. In Section 2, a brief review of the material models and the simulation method is given. In Section 3, the detailed simulation results by using the developed method are presented, including the binder molecule conformations on the polymer surface; the binding affinity analysis and the temperature effect. The main results are summarized in Section 4.

\section{Materials and methods}

\subsection{Binder molecules and polymer film}

The five small molecules studied in this work are Eugenol, Dlimonene and the three aldehydes - C8, C9 and C10. They are frequently used in the food industry to obtain products with different tastes. Their bindings with the lining polymeric films often occur during food products storage. These five molecules are selected based on differences in their molecular structures, functional groups and polarities (see Table 1) to test the capability of the developed binding affinity assessment method. As shown in Fig. 1, the three aldehydes have a straight chain structure with the $-\mathrm{CH}=\mathrm{O}$ group at one end of the chain, and only differ in the chain length. Unlike the aldehydes, both Eugenol and D-limonene have a ring structure. They have different hydrophobicity which is reflected in the $\log P$ values listed in Table 1 with a higher value of $\log P$ implying increased hydrophobicity.

The model polymer film used in the simulations is prepared by mixing prescribed proportions of the following four components: methyl acrylate, poly(ethylene-co-acrylic acid) (EAA), hydrogen 
Table 1

Physicochemical characteristics of the five binder molecules studied.

\begin{tabular}{|c|c|c|c|c|c|}
\hline Flavor compounds (formula) & $\mathrm{MW}^{\mathrm{a}}(\mathrm{g} / \mathrm{mol})$ & Melting point $\left({ }^{\circ} \mathrm{C}\right)$ & Density $^{\mathrm{b}}\left(\mathrm{g} \mathrm{cm}^{-3}\right)$ & Solubility in water at $25^{\circ} \mathrm{C}^{\mathrm{c}}(\mathrm{mg} / \mathrm{L})$ & $\log P^{\mathrm{d}}$ \\
\hline Octanal $\left(\mathrm{C}_{8} \mathrm{H}_{16} \mathrm{O}\right)$ & 128.21 & -23 & 0.821 & 560 & 2.78 \\
\hline Nonanal $\left(\mathrm{C}_{9} \mathrm{H}_{18} \mathrm{O}\right)$ & 142.24 & -19.3 & 0.826 & 96 & 3.27 \\
\hline Decanal $\left(\mathrm{C}_{10} \mathrm{H}_{20} \mathrm{O}\right)$ & 156.27 & -3.9 & 0.830 & 15.6 & 3.76 \\
\hline Eugenol $\left(\mathrm{C}_{12} \mathrm{H}_{12} \mathrm{O}_{2}\right)$ & 164.20 & -9.2 & 1.065 & 2463 & 2.49 \\
\hline D-Limonene $\left(\mathrm{C}_{10} \mathrm{H}_{16}\right)$ & 136.23 & -74.3 & 0.841 & 13.8 & 4.57 \\
\hline
\end{tabular}

Obtained from PubChem (https://pubchem.ncbi.nlm.nih.gov).

a Molecular weight.

b Values at $20^{\circ} \mathrm{C}$.

c Obtained from Handbook of Aqueous Solubility Data (2nd edition).

d Values of $\log K_{o w}$, partition coefficient between n-octanol and water.

(a)<smiles>CCCCCCCC=O</smiles>

(b)<smiles>CCCCCCCCC=O</smiles>

(c)<smiles>CCCCCCCCCC=O</smiles>

(d)

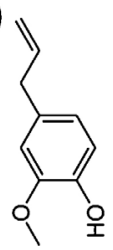

(e)

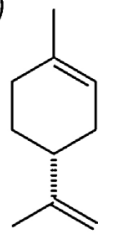

Fig. 1. Molecular structures of (a) C8; (b) C9; (c)C10; (d) Eugenol and (e) D-limonene.

peroxide $\left(\mathrm{H}_{2} \mathrm{O}_{2}\right)$ and poly(ethyleneimine). These four components are randomly connected to each other through a complex polymerization process to form a highly crosslinked and amorphous network structure. Thus it is very difficult to evaluate the binding affinity through the solubility parameters. Based on the analysis of possible reactions occurring in the mixture, it is assumed that the final heterogeneous polymer film is mainly made of poly(methyl acrylate) (PMA), the EAA, and the linear and the branched poly(ethyleneimine) (LPEI and BPEI) polymers. Their chemical structures are illustrated in Fig. 2.

\subsection{Atomistic models}

Due to the complex polymerization process of preparing the film it is very challenging to construct an atomistic model that accurately describes the film system. However, it is noted that the adsorption of small molecules occurs mainly at the film surface, a process that is not significantly affected by the film's interior structure. Thus, the simulated film is prepared by directly mixing the four polymers without modeling crosslinking. By investigating these binder-film interactions, deep insights into the binding of the small molecules with the polymer film can still be gained. Note that the cross-linking algorithms to construct atomistic models with complex network structures are available in the literature if the curing reaction mechanisms are known $[27,28]$.

The atomistic model for the binding affinity analysis consists of a slab of the polymer film and a water box containing a single binder molecule. In Fig. 3 is shown the assembled atomistic model of the binder-film system. The polymer film is prepared by randomly mixing 12 PMA chains with 20 repeating units $(n=20), 17$ EAA chains with 3 repeating monomers $(n=3)$ of 14 mers ethylene $(x=14)$ and 1 mer acrylic $(y=1), 1$ LPEI chain with 10 repeating units and 1 BPEI chain with only 1 repeating unit $(n=1)$. Curing reactions among these polymers are not considered. The lengths of these polymer chains are chosen to reflect their proportion in the film. The preparation of the polymer film involves the process of densification. Here we added two impenetrable walls on either side of the box in the $z$-direction of the mixed polymer system to compress the film to its experimental density value of $1.01 \mathrm{~g} \mathrm{~cm}^{-3}$ at $300 \mathrm{~K}$. Keeping the two walls fixed, the compressed film was annealed to release internal stresses and relax the system to surface-like chains. Subsequently the upper wall was removed and the film surface was further relaxed to minimize the surface energy for the binding affinity analysis (Fig. 3a). The prepared polymer film measured $5.2 \mathrm{~nm} \times 4.2 \mathrm{~nm} \times 3.5 \mathrm{~nm}$ and had 9572 atoms with a mass density of $0.968 \mathrm{~g} \mathrm{~cm}^{-3}$ in its central part at $300 \mathrm{~K}$. This mass density is slightly below the experimental value, which is not surprising since the crosslinking reactions that have a volume shrinkage effect are not considered in the film preparation. Even though the bottom wall was kept fixed during the relaxation of the film, it will not affect the subsequent binding free energy calculation since the thickness of the film prevented it from interacting with the small molecules.

The periodic water box was prepared by using the surface dimensions of the polymer film; 4240 water molecules were packed in the $5.2 \mathrm{~nm} \times 4.2 \mathrm{~nm} \times 4.35 \mathrm{~nm}$ box to achieve the mass density of $0.99 \mathrm{~g} \mathrm{~cm}^{-3}$. The single binder molecule (Fig. 1) was then inserted near the center of the interface between the water box and the polymer film slab to facilitate its adsorption. An impenetrable wall was added to the top of the water box to prevent evaporation of water molecules in subsequent simulations and was placed $20 \AA$ from the top of the water layer, which is larger than the cut-off distance for interaction between atoms in the MD simulation. Periodic boundary conditions were applied in all three directions of the assembled atomistic model. The surface dimensions of the polymer film substrate are large enough to prevent the single binder molecule (Fig. 3c) from interacting with its images.

\subsection{Metadynamics method}

The Metadynamics method developed by Laio and Parrinello [24] is employed to calculate the adsorption free energy of binder molecules and evaluate the binding sensitivity of the polymeric film. This technique calculates the free energy profile (FEP) of certain chemical or physical processes along the reaction coordinates of interest. It involves adding a series of Gaussian potentials to overcome significant energy barriers between different states and drive the system along the reaction coordinates. By tracking and summing the added Gaussian potentials, the FEP is reconstructed and
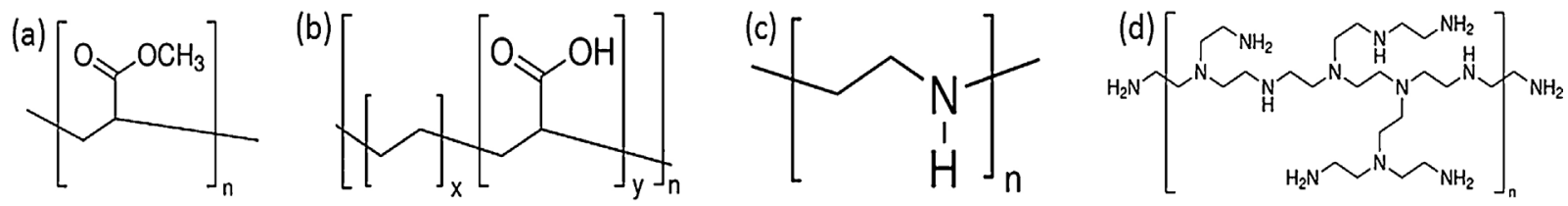

Fig. 2. Chemical structures of (a) PMA; (b) EAA; (c) LPEI and (d) BPEI. 
(a)

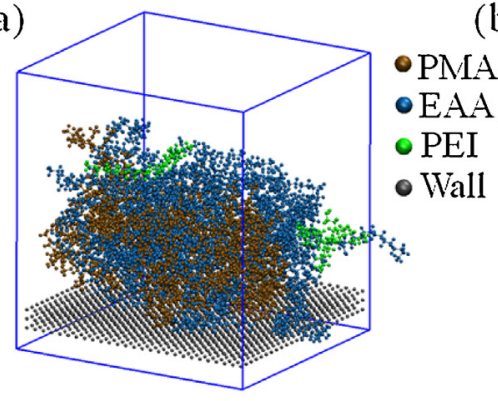

(b)

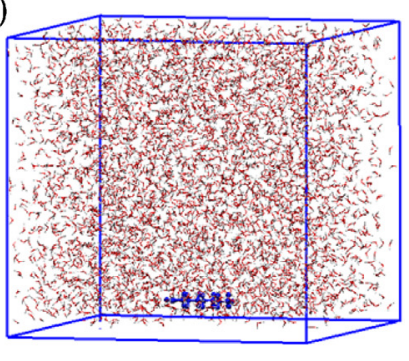

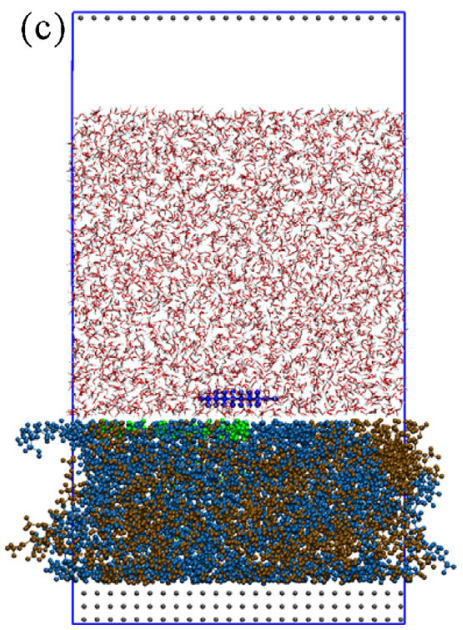

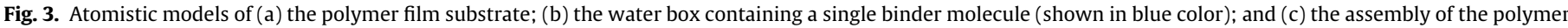

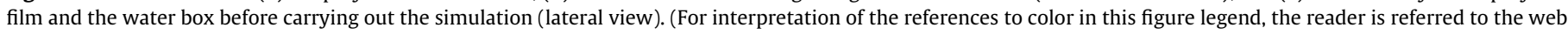
version of the article.)

the corresponding free energy barriers to move the system from one state to the other state are estimated. The reaction coordinates that describe the chemical or physical processes are often referred to as collective variables or CVs. Let $s(x)=\left(s_{1}(x), \ldots, s_{d}(x)\right)$ be the $d$ collective variables and $x$ be system's degrees of freedom with a restricted number $n$ ( $n \leq 3 N ; N$ is the total number of atoms). The Gaussian potential deposited into the system at time $t$ is defined as

$$
\begin{gathered}
V(s(x), t)=\omega \sum_{t^{\prime}=\tau_{G}, 2 \tau_{G}, \ldots} \exp \left(-\sum_{i=1}^{d} \frac{\left[s_{i}(x)-s_{i}\left(x\left(t^{\prime}\right)\right)\right]^{2}}{2 \sigma_{i}^{2}}\right) \\
t^{\prime}<t
\end{gathered}
$$

where $\omega$ is the Gaussian height, $\sigma_{i}$ the Gaussian width of the $i$-th CV, and $\tau_{G}$ the frequency at which the Gaussian potential is deposited. With this additional potential, the biased forces applied on atoms or molecules to facilitate the system in moving along the CVs can be written as

$$
-\frac{\partial V(s, t)}{\partial x}=-\sum_{i=1}^{d} \frac{\partial V(s, t)}{\partial s_{i}} \frac{\partial s_{i}(x)}{\partial x}
$$

Each time the Gaussian potential is deposited into the system, the corresponding CVs are varied under the effect of the biased forces. After the deposition of a certain number of Gaussian potentials, the potentials associated with different values of CVs can be identified. The free energy versus CVs (or FEP) at that time can be reconstructed through the summation of the identified potentials. The FEP eventually converges with increase in time as more potentials are deposited and identified.

Both the Gaussian potential parameters and the CVs significantly influence the accuracy and the efficiency of the free energy reconstruction in Metadynamics simulations [29]. To investigate their influences, two kinds of CVs are used and compared in the current binder-polymer interaction simulations (Fig. 3c): the first $\mathrm{CV}$ (CV1) is taken as the distance between the centers of mass (CM) of the binder molecule and of the polymeric film, while the second $\mathrm{CV}(\mathrm{CV} 2)$ is chosen as the distance of the binder molecule CM from the polymer surface. For CV1, the Gaussian potentials with height $0.05 \mathrm{~kJ} \mathrm{~mol}^{-1}$ and width $0.35 \AA$ are deposited every $0.1 \mathrm{ps}$ to reconstruct the FEP of the binder-polymer system from the initial attached state to a detached state. While for CV2, the finer Gaussian potentials with height $0.01 \mathrm{~kJ} \mathrm{~mol}^{-1}$ and width $0.035 \AA$ are used to reconstruct the FEP. They are also deposited every $0.1 \mathrm{ps}$ in the system. With these finer Gaussian parameters, more details of the FEP can be explored.

\subsection{Simulation details}

The OPLS-aa force field [30] is used to describe all the interand intra-molecule interactions in the binder-polymer system. By employing this nonreactive force field, it is understood that the partial charges of all atoms in the system do not change throughout the simulation, and the binder-polymer binding process is dominated by the weak bonds rather than by the covalent interactions. The water molecules in the system are described by the TIP3P model with a cutoff distance of $10 \AA \AA$ [31]. The SHAKE algorithm [32] is used to fix the bond lengths and angles of TIP3P water molecules during the simulations. The van der Waals (vdW) and the short-range Coulombic interactions are truncated at a cutoff distance of $10 \AA$, while the long-range Coulombic interaction is calculated with the PPPM solver [33]. The assembled binder-polymer system (Fig. 3c) is first equilibrated in NVT ensemble at $300 \mathrm{~K}$ for $1.0 \mathrm{~ns}$ with a time step of $1 \mathrm{fs}$ to achieve the local lowest energy configuration. After that, Metadynamics simulations are performed for $2.0 \mathrm{~ns}$ by using the adjusted Gaussian parameters and the chosen CVs. All Gaussian potentials deposited during the $2.0 \mathrm{~ns}$ of simulation are collected and used to reconstruct the FEP. All simulations are carried out using the MD package LAMMPS [34] with the PLUMED [29] plug-in for the Metadynamics analysis.

\section{Results and discussion}

In Metadynamics simulations, the FEP of the binder-polymer system from an absorbed state to a desorbed state is reconstructed to determine the binding affinity. The conformations of binder molecules at the polymer surface that define the absorbed state are thus of great importance. In Fig. 4 we have shown the equilibrated conformations of the five small binder molecules studied in this work after $1.0 \mathrm{~ns}$ of standard MD simulation under the NVT ensemble. The binder molecules initially are inserted in water solvent near the center of the polymer surface to facilitate the absorption process (see Fig. 3c). Under effects of van der Waals and Coulombic interactions, all five binder molecules are observed to be well attached to the polymer surface and no water molecules can be seen in the gap between the binder molecules and the polymer film. The aldehyde molecules (C8, C9 and C10) show an extensive structure and align parallel with the polymer surface 


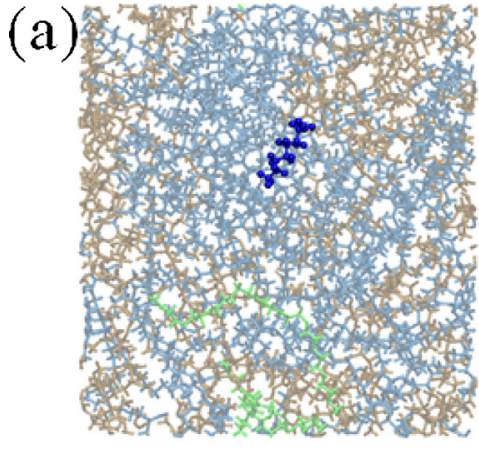

(d) (b)

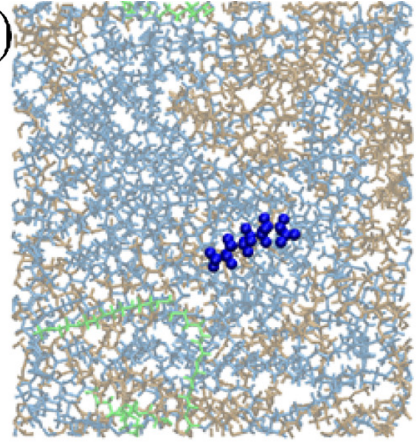

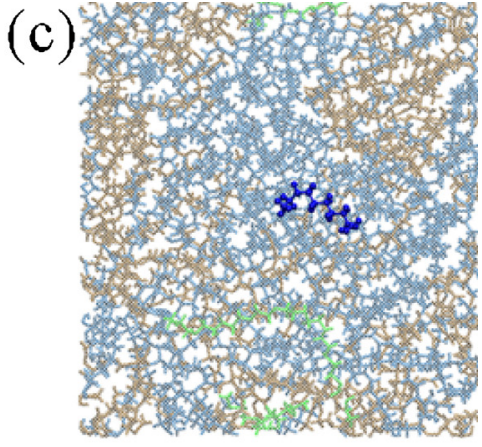

(e)
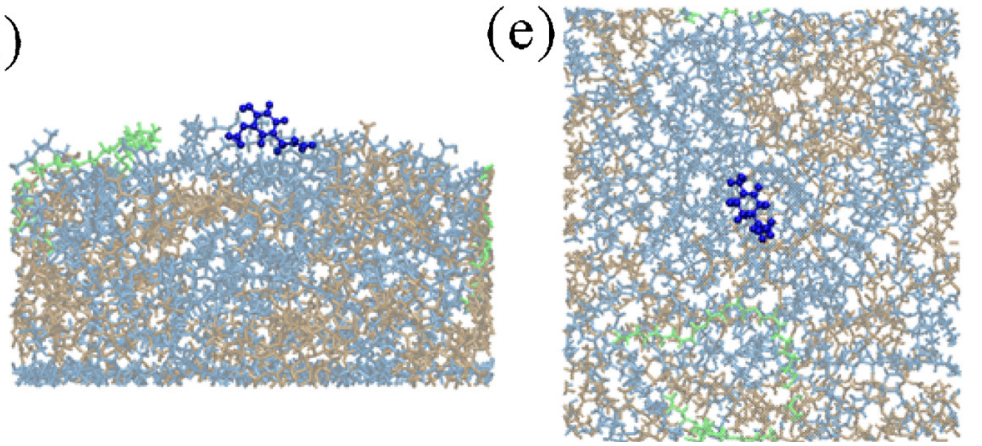

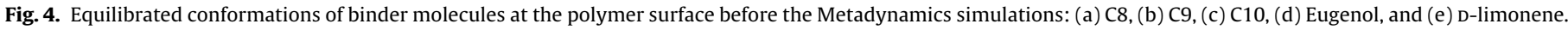

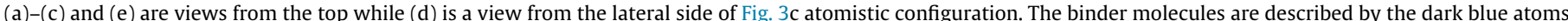

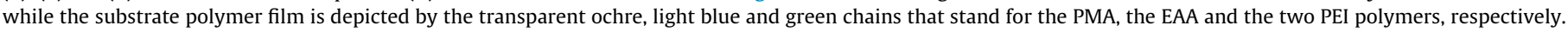
The water molecules are omitted for clarity. (For interpretation of the references to color in this figure legend, the reader is referred to the web version of the article.)

as all their functional groups are involved in the binding process (Fig. 4a-c). The D-limonene molecule shows a similar phenomenon as its ring-structure plane also aligns parallel with the polymer surface (Fig. 4e) forming a flat binding mode. On the contrary, the Eugenol molecule tends to align perpendicular to the polymer surface (Fig. 4d). Further analysis of the binding groups reveals that the hydroxyl group that interacts strongly with the water molecules keeps the Eugenol molecule from lying down on the polymer surface. This strong interaction between the Eugenol and water molecules is also reflected in its solubility and $\log P$ values in water. As can be seen from values listed in Table 1, the Eugenol is most soluble in water and least hydrophobic among the five small molecules studied.

We also investigate the time evolution of the CM displacement and the radius of gyration $\left(R_{g}\right)$ for the binder compounds during the equilibrium process to check the stability of the equilibrated conformations, as illustrated in Fig. 5. Here $R_{g}$ is defined as:

$R_{g}=\frac{1}{N}\left(\sum_{i=1}^{N}\left(r_{i}(t)-r_{c m}(t)\right)^{2}\right)^{1 / 2}$

where $N$ is the number of atoms in the binder molecule; $r_{i}(t)$ and $r_{c m}(t)$ are, respectively, the position vectors of each atom in the binder molecule and the CM for the whole molecule at time $t$. The last $0.5 \mathrm{~ns}$ equilibrium simulation is used for analyzing these since the binder molecules have already been adsorbed on the polymer surface. Thus, the CM displacements of the binder molecules are measured relative to the original coordinates at $t=0.5 \mathrm{~ns}$. As shown in Fig. 5a, though there is a sudden movement of the C8 and the D-limonene molecules between $t=0.6 \mathrm{~ns}$ and $t=0.65 \mathrm{~ns}$, all five binder molecules reach their stable conformations on the polymeric film surface before the start of the Metadynamics simulations. These stable conformations of binder molecules can also be seen in the time history of $R_{g}$ (Fig. 5b). Generally, an increase of $R_{g}$ represents the unfolding of molecules to an extended structure, while a decrease of $R_{g}$ means the molecules coil and collapse to a compact structure. This is particularly true for those molecules with long chain structure. As seen in Fig. 5b, though the variation of $R_{g}$ for aldehyde molecules is greater than those of the Eugenol and the D-limonene molecules with ring structure, the relatively small variation values suggest that all five binder molecules reach the stable adsorption states on the polymer surface.

In Fig. 6 we have plotted the evolution of the CM distances between the binder molecules and the polymer film during the equilibrium process. It is clear that the $\mathrm{CM}$ distances for all five binder molecules are relatively stable beyond the simulation time of $0.65 \mathrm{~ns}$, which confirms again stable adsorption states have been obtained. These $\mathrm{CM}$ distances are slightly different for each binder molecule with the averaged values of $20.21 \AA, 18.62 \AA, 20.70 \AA$, $18.21 \AA$ and $18.74 \AA$ for the $\mathrm{C} 8$, the $\mathrm{C} 9$, the $\mathrm{C} 10$, the Eugenol and the D-limonene, respectively. This CM distance quantifies the binding distance of binders on the rough surface of the polymeric film (see Fig. 4d) and thus is chosen to be the CV1 in subsequent Metadynamics simulations. To study the influences of the chosen CVs on the FEP reconstruction, we also take the $\mathrm{CM}$ distance of the binder molecules from the polymer surface as CV2 in the simulations. Due to the roughness of the polymer surface, the CV2 is defined based on the absolute positions of the CM distance of the binder molecules, rather than as the relative distance between the binder and the polymer surface. Over the course of Metadynamics simulations, both CVs span at least a distance of $35 \AA$ for all binder-polymer systems explored, which can well distinguish between the adsorbed and the desorbed states of binder molecules.

The typical Metadynamics simulation described in Section 2 is illustrated by using the C9-polymer system at $T=300 \mathrm{~K}$ as a representative example. During the simulation, the desorbing behaviors of the C9 molecule from the polymer surface as a result of the Gaussian potentials deposition on CV1 are displayed in Fig. 7. The corresponding FEPs along the CV1 are reconstructed at different 

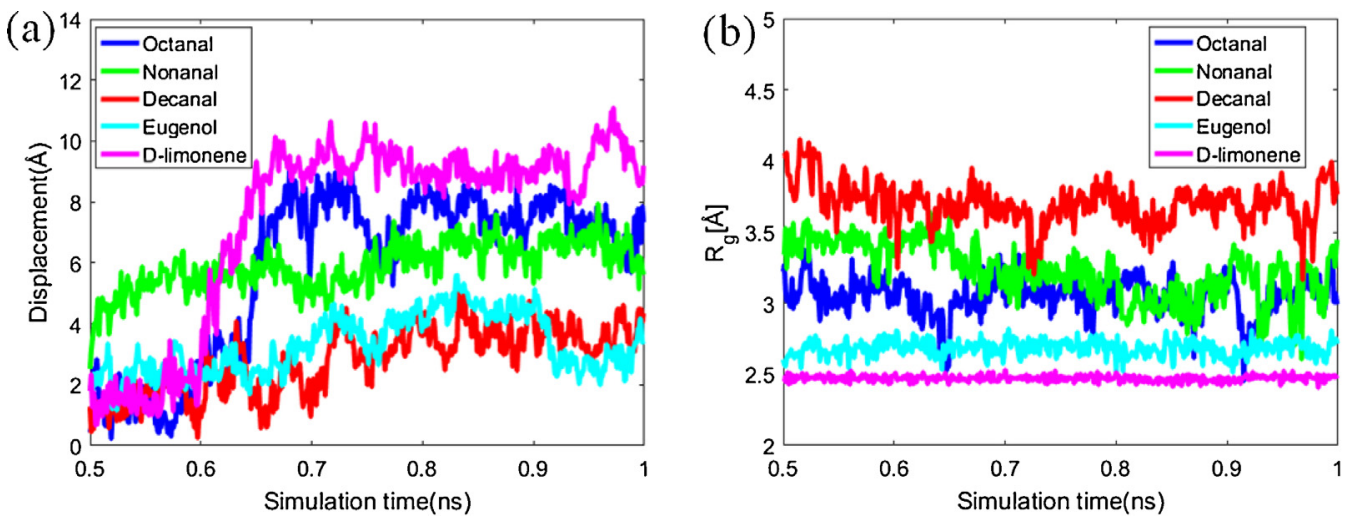

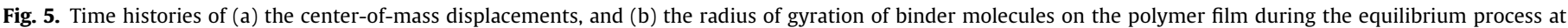
$T=300 \mathrm{~K}$.

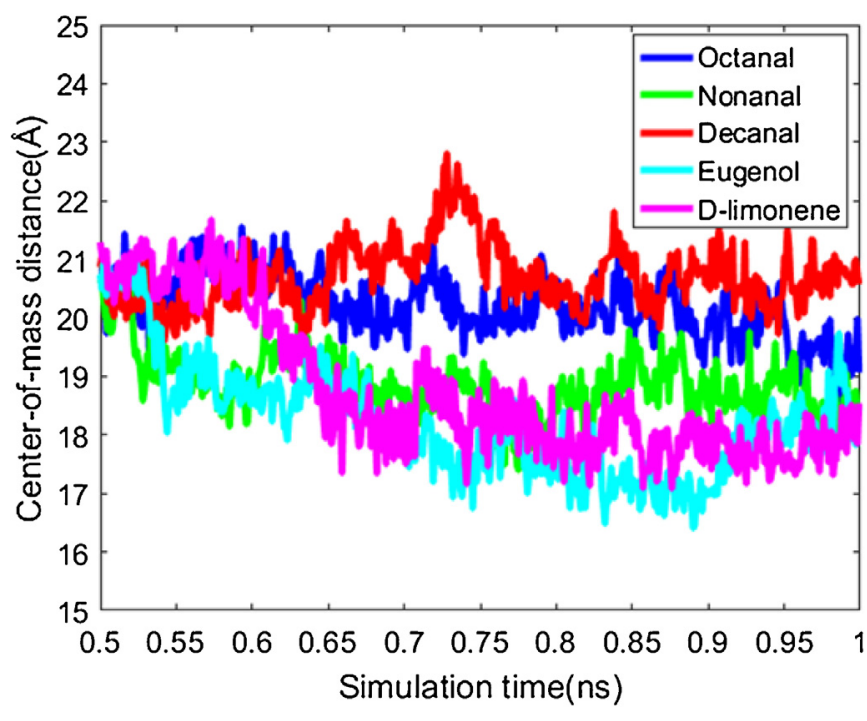

Fig. 6. Time histories of the center-of-mass distances between the binder molecules and the polymeric film during the equilibrium process at $T=300 \mathrm{~K}$.

simulation times and plotted in Fig. 8a. From the reconstructed FEPs, it can be seen that the well depth of the free energy increases with an increase in the simulation time as more Gaussian potentials are deposited. The bottom black curve shows the convergence of the FEPs after $t>0.8 \mathrm{~ns}$, which describes the physical energy landscapes of the adsorption as a function of the CM distance between the C9 molecule and the polymeric film substrate. The convergence of the FEPs also implies that the C9 molecule detaches at $t=0.6-0.8 \mathrm{~ns}$, which can be more clearly seen from the snapshots displayed in Fig. 7b-d. The strongest binding occurs when the distance between the CM of the C9 molecule and the polymer film equals $1.60 \mathrm{~nm}$, which leads to the minimum free energy with a well depth of $-322.82 \mathrm{~kJ} \mathrm{~mol}^{-1}$. This negative value of the well depth suggests that the adsorption of the C9 molecule onto the polymer surface is spontaneous and thermodynamically favorable. From the corresponding microstructure shown in Fig. 7a, it is observed that the oxygen atom in the C9 molecule closely interacts with the acrylic acid group of the EAA component in the polymeric film, which results in the large well depth in the FEPs. Subsequently, there are several intermediate stages (Fig. 7b-d) before the C9 molecule is fully desorbed. From Fig. $7 \mathrm{~b}$ and c, it can be seen that the C9 molecule starts to desorb after a certain amount of Gaussian energy has been added to the system. However, the interfacial adhesion remains at a high level due to the interactions between the functional groups as mentioned above. From Fig. 7b to d, it can be seen that the C9 molecule continuously moves away from the polymer film substrate as more Gaussian potentials are deposited. Eventually, the C9 molecule is fully desorbed from the substrate, implying the disappearance of the van der Waals and the Coulomb interactions between these two materials and the completion of the detachment. Furthermore, the sequential detachment processes also demonstrate that the C9 molecule is biased to escape from the local free energy minimum in a non-collective manner and the sampling time is long enough to allow the actual FEP to be filled up by the chosen Gaussian potentials. Due to the constant Gaussian deposition rate, the system then eventually explores the entire CV1 domain with equal probability before the detachment is completed.

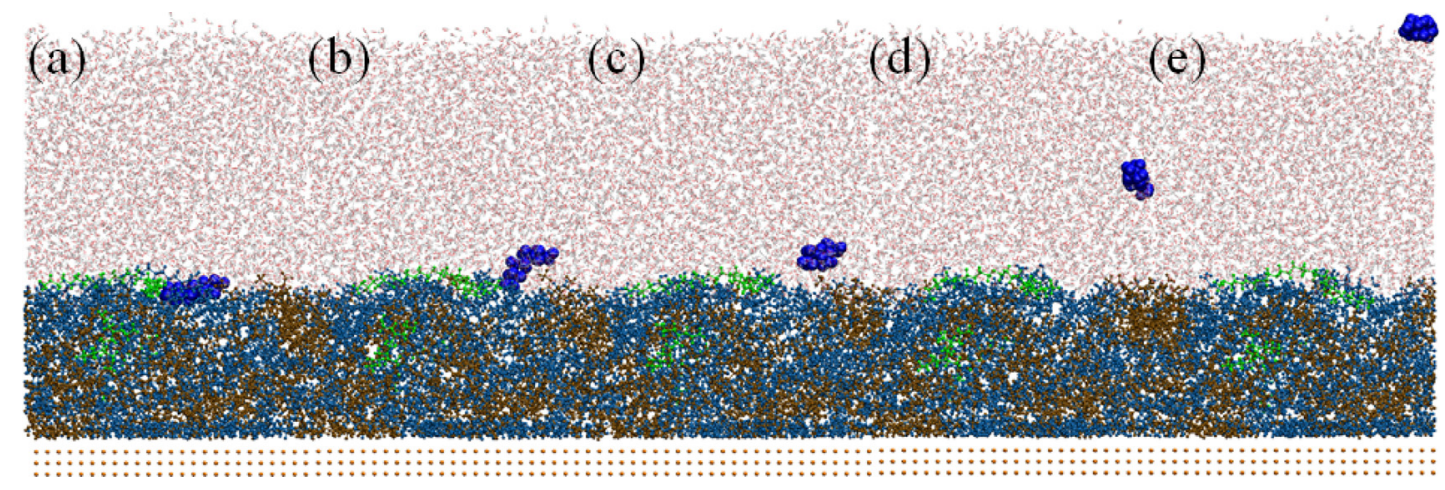

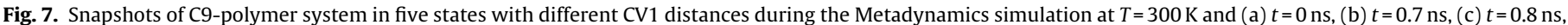

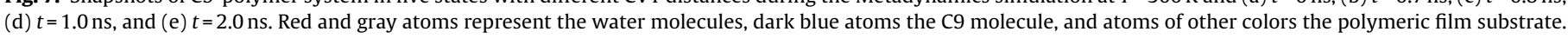
(For interpretation of the references to color in this figure legend, the reader is referred to the web version of the article.) 

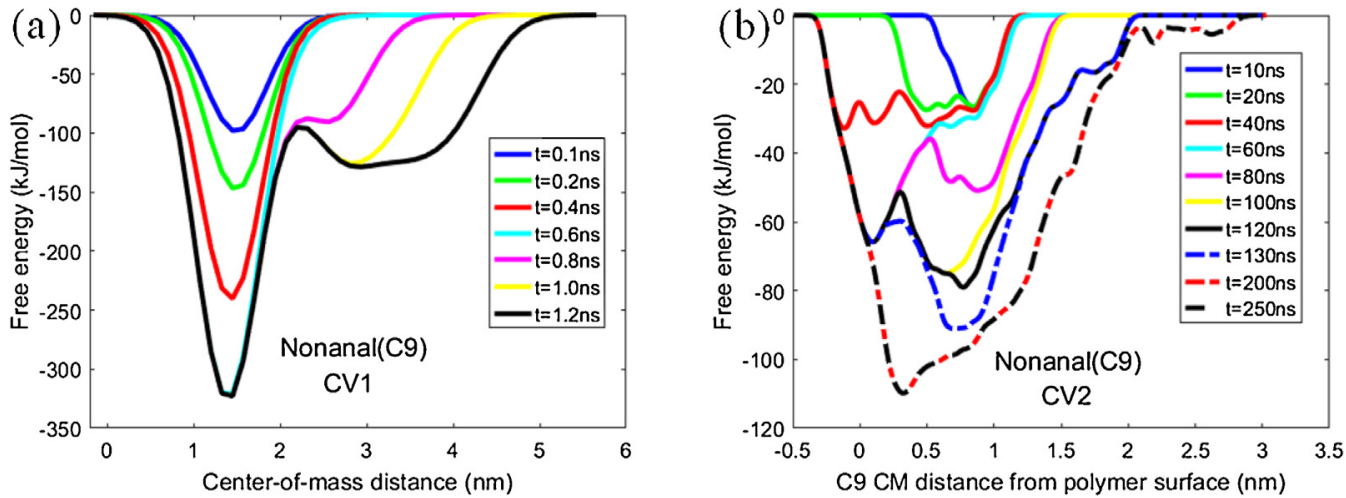

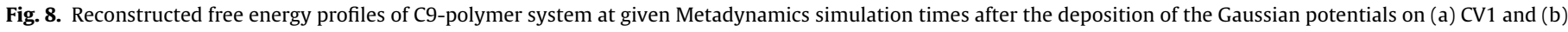
CV2 at $T=300 \mathrm{~K}$. Letters a, $\mathrm{d}$ and e denote the corresponding three states of the C9-polymer system depicted in Fig. 7.

Table 2

Binding free energies between the small molecules and the polymer film $\left(\mathrm{kJ} \mathrm{mol}^{-1}\right)$.

\begin{tabular}{llllll}
\hline Temperature & C8 & C9 & C10 & Eugenol & D-Limonene \\
\hline $300 \mathrm{~K}(\mathrm{CV} 1)$ & -343.17 & -322.82 & -360.91 & -181.77 & -130.62 \\
$300 \mathrm{~K}(\mathrm{CV} 2)$ & -109.12 & -109.82 & -134.39 & -75.74 & -81.09 \\
$277 \mathrm{~K}(\mathrm{CV} 1)$ & -172.27 & -204.58 & -278.87 & -165.77 & -108.40 \\
$277 \mathrm{~K}(\mathrm{CV} 2)$ & -82.36 & -79.16 & -76.92 & -70.23 & -74.86 \\
\hline
\end{tabular}

These observations imply that our selection of Gaussian parameters for the Metadynamics sampling is reasonable.

To further understand the influence of the Gaussian potential parameters and the CVs on the accuracy and efficiency of the binding free energy calculation, the finer Gaussian potentials with height $0.01 \mathrm{~kJ} \mathrm{~mol}^{-1}$ and width $0.035 \AA$ and $\mathrm{CV} 2$ are also used in the Metadynamics simulations to reconstruct the FEPs. The results are plotted in Fig. 8b for the C9-polymer system. The reconstructed FEPs in the CV2 simulations are more structured than those in the CV1 simulations. The finer Gaussians allow a more detailed exploration of the entire CV2 domain, but with the compromise of the computational efficiency. As shown in Fig. 8, it takes $200 \mathrm{~ns}$ simulation time for the CV2 simulation to converge and reach the minimum free energy, while the CV1 simulation only takes $1.2 \mathrm{~ns}$. The converged minimum free energy for the CV2 simulation is about $-109.82 \mathrm{~kJ} \mathrm{~mol}^{-1}$, which is only one-third of that in the CV1 simulation. This is expected since a portion of the deposited Gaussian energies is also used to bias the degrees of freedom of the polymer film in the CV1 simulations (see Eq. (5)). Therefore, more Gaussian energies are needed to detach the C9 molecules from the polymer surface in the CV1 simulations and the reconstructed FEPs are correspondingly deeper than those in the CV2 simulations. Thus all Gaussian potentials are used to bias the C9 molecule in the CV2 simulations, and the C9-polymer binding free energy can be more properly calculated from the well depth of the corresponding FEPs.

The Metadynamics simulations of the $\mathrm{C} 8$, the $\mathrm{C} 10$, the Eugenol and the D-limonene molecules on the polymeric film show a similar desorption process as that observed for the C9 case: starting from adsorption, going through intermediate states and finally achieving the desorption. In Fig. 9 we have displayed the reconstructed FEPs along the CV1 and the CV2 for these four binder compounds; the calculated binding free energies are summarized in Table 2 . The FEPs of the $\mathrm{C} 8$ and the $\mathrm{C} 10$ molecules are quite similar to those of the C9 molecule for both the CV1 and the CV2 since the three molecules share a very similar structure with the only difference being in the carbon chain length. In the CV1 simulations, the minimum free energies of the C8- and the C10-polymer systems appear at the CV distances of $20.8 \AA$ and $20.5 \AA$, respectively, which are related to their stable CM distances after the equilibrium process (Fig. 6). The corresponding free energy barriers between the adsorbed and the desorbed states are $-343.17 \mathrm{~kJ} \mathrm{~mol}^{-1}$ and $-360.91 \mathrm{~kJ} \mathrm{~mol}^{-1}$, which are close to that for the C9 case (Fig. 8a). In the CV2 simulations, it can be seen that the minimum free energy appears right at the C8- and the C10-polymer interfaces, with a CV distance of $\sim 5 \AA$ due to the roughness of the polymer surface. The calculated binding free energies, $-109.12 \mathrm{~kJ} \mathrm{~mol}^{-1}$ and $-134.39 \mathrm{~kJ} \mathrm{~mol}^{-1}$ are also close to that for the C9 case (Fig. 8b). The FEPs of the Eugenol and the D-limonene molecules are different from those of the aldehyde molecules (Fig. 9e and h). The lowest well depth regions of the free energy wells in these two cases are wider than that for the aldehyde molecules, and span a CV distance of larger than $5 \AA$. These wider FEPs might be related to the ring structure of the Eugenol and the D-limonene molecules, which allows them to possess a larger number of conformation states at the polymer surface than that of the aldehyde molecules with chain structures. The FEP well depths for the Eugenol and the D-limonene molecules are much smaller than that for the aldehyde cases. In the CV1 simulations, the corresponding largest free energy barriers are $-181.77 \mathrm{~kJ} \mathrm{~mol}^{-1}$ and $-130.62 \mathrm{~kJ} \mathrm{~mol}^{-1}$, respectively, for the Eugenol and the D-limonene molecules, while their respective values are $-75.74 \mathrm{~kJ} \mathrm{~mol}^{-1}$ and $-81.09 \mathrm{~kJ} \mathrm{~mol}^{-1}$ in the CV2 simulations. Since a higher negative free energy barrier means that the adsorption is more likely to occur, it can thus be inferred from both the CV1 and the CV2 simulations that the studied polymer film has a higher affinity for the aldehydes than that for the Eugenol and the D-limonene.

Another advantage of the Metadynamics analysis is that simulations can be readily performed at various temperatures. It can potentially overcome limitations of the experimental methods that are typically restricted to the room temperature. The Metadynamics simulations of the five binder-polymer systems were performed at $277 \mathrm{~K}$ to study the temperature effect. In Table 2, we have summarized the binding free energies calculated from the reconstructed FEPs along both the CV1 and the CV2 for the five binder molecules. It can be seen that the binding free energy decreases with the decrease of temperature for both the CV1 and the CV2 simulations, indicating the reduced binding affinity between the binder molecules and the polymer film. However, the temperature effect is not the same among the five binders. The aldehyde molecules are more sensitive to the temperature change than both the Eugenol and the D-limonene molecules. This reduction of binding free energy at lower temperature can be understood from the definition of the Gibbs free energy (see Eq. (3)). The entropy term $(T \Delta S)$ to drain water molecules away from the polymer surface generally decreases with the decrease of temperature. Thus the adsorption process also becomes less favorable as the temperature decreases. 

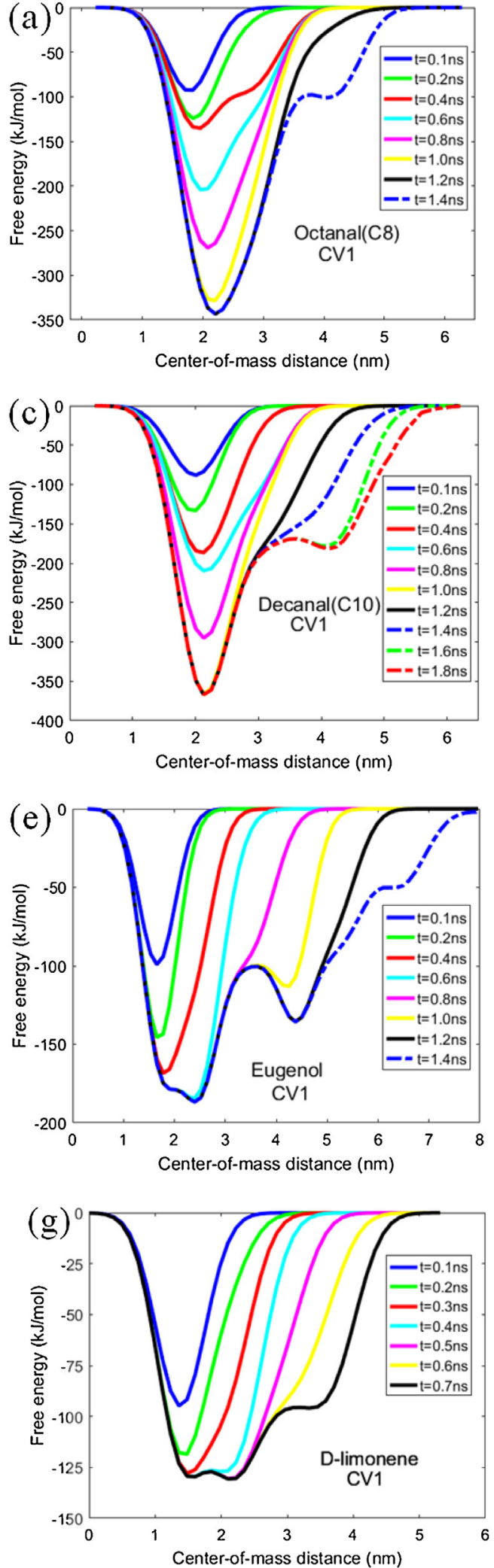
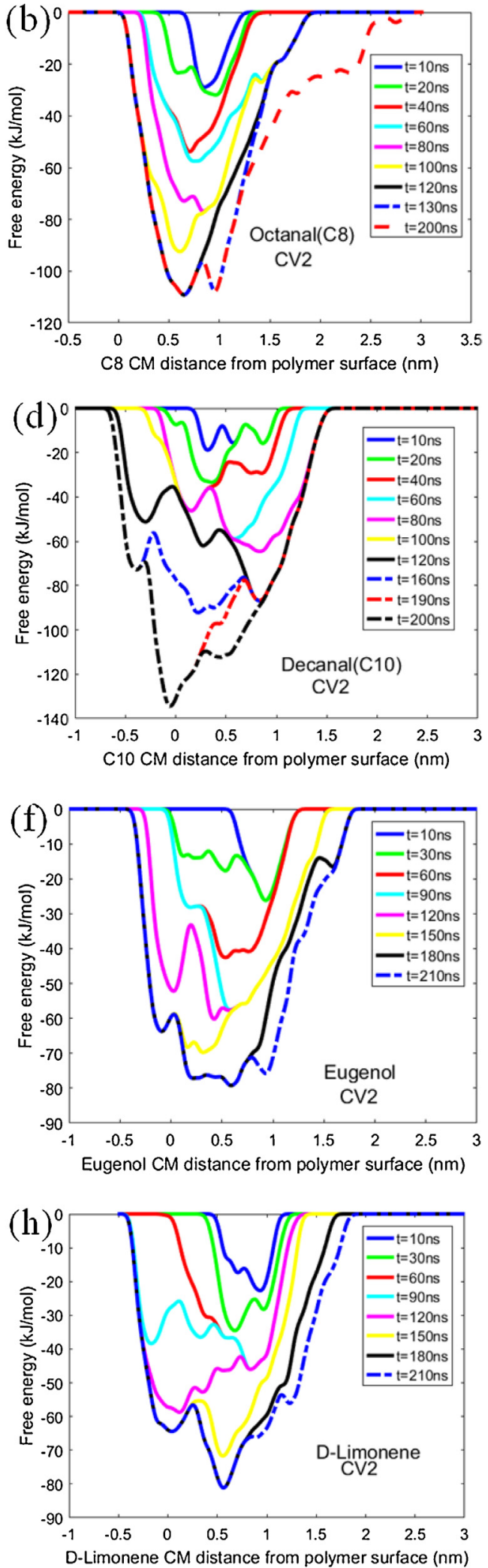

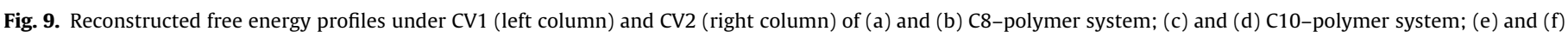
Eugenol-polymer system, and (g) and (h) D-limonene-polymer system at different Metadynamics simulation times and $T=300 \mathrm{~K}$; CM stands for the center of mass.

\section{Conclusions}

By using the standard molecular dynamics and the Metadynamics methods with two kinds of collective variables (CVs), we have studied the adsorption of the five molecules, C8, C9, C10, Eugenol and D-limonene, onto a model polymeric film and have successfully computed the corresponding binding free energies. Even though the choice of CVs affects the accuracy of the calculated binding free energy for a specific binder-polymer system, it does not affect the computed affinity of the binder molecules to the polymer film. Our 
simulations also show that the adsorption of binder molecules onto the polymer surface is a spontaneous process with a flat binding conformation. The aldehyde and the D-limonene molecules generally align parallel to the polymer surface whereas the Eugenol molecules tend to align perpendicular to the polymer surface due to their interactions with water molecules. The spontaneous adsorption of the binder molecules by the polymeric film is driven by the negative nature of the binding free energy. The binding free energies of the aldehyde molecules are found to be higher than that of the Eugenol and the D-limonene molecule, indicating the high affinity of the aldehyde molecules to the polymer film. The temperature generally has an enhanced effect on the binder molecule adsorption since it is found that the binding free energy increases with the increase in temperature. However, the temperature effect is not the same for the five molecules. It affects more significantly the adsorption of the aldehyde molecules than that of the Eugenol and the D-limonene molecules. Even though the proposed method provides only a qualitative prediction of the small molecule absorption by comparing the binding free energies between different binder compounds and the film, it is potentially very useful for the selection and the design of polymeric barrier films, especially for cases when the polymers have a complicated microstructure.

\section{References}

[1] M.G. Sajilata, K. Savitha, R.S. Singhal, V.R. Kanetkar, Scalping of flavors in packaged foods, Compr. Rev. Food Sci. Food Saf. 6 (1) (2007) 17-35.

[2] A. Peychès-Bach, M. Moutounet, S. Peyron, P. Chalier, Factors determining the transport coefficients of aroma compounds through polyethylene films, J. Food Eng. 95 (1) (2009) 45-53.

[3] C. Dury-Brun, Y. Hirata, V. Guillard, V. Ducruet, P. Chalier, A. Voilley, Ethyl hexanoate transfer in paper and plastic food packaging by sorption and permeation experiments, J. Food Eng. 89 (2) (2008) 217-226.

[4] D. Cava, R. Catala, R. Gavara, J.M. Lagaron, Testing limonene diffusion through food contact polyethylene by FT-IR spectroscopy: film thickness, permeant concentration and outer medium effects, Polym. Test. 24 (4) (2005) 483-489.

[5] R. Kühne, R.U. Ebert, F. Kleint, G. Schmidt, G. Schüürmann, Group contribution methods to estimate water solubility of organic chemicals, Chemosphere 30 (11) (1995) 2061-2077.

[6] E. Stefanis, C. Panayiotou, Prediction of Hansen solubility parameters with a new group-contribution method, Int. J. Thermophys. 29 (2) (2008) 568-585.

[7] D.W. Van Krevelen, Properties of Polymer: Their Estimation and Correlation with Chemical Structure, 2nd ed., Elsevier, Amsterdam, 1976.

[8] A.R. Berens, The solubility of vinyl chloride in poly(vinyl chloride), Angew. Makromol. Chem. 47 (1) (1975) 97-110.

[9] Y. Kamiya, T. Hirose, K. Mizoguchi, Y. Naito, Gravimetric study of high-pressure sorption of gases in polymers, J. Polym. Sci. B: Polym. Phys. 24 (7) (1986) 1525-1539.

[10] G. DiPaola-Baranyi, Estimation of polymer solubility parameters by inverse gas chromatography, Macromolecules 15 (2) (1982) 622-624.

[11] R. Gavara, R. Catalá, P. Hernández-Muñoz, Study of aroma scalping through thermosealable polymers used in food packaging by inverse gas chromatography, Food Addit. Contam. 14 (6-7) (1997) 609-616

[12] D. Cava, J.M. Lagarón, A. López-Rubio, R. Catalá, R. Gavara, On the applicability of FT-IR spectroscopy to test aroma transport properties in polymer films, Polym. Test. 23 (5) (2004) 551-557.
[13] X. Chen, C. Yuan, C.K.Y. Wong, G. Zhang, Molecular modeling of temperature dependence of solubility parameters for amorphous polymers, J. Mol. Model. 18 (6) (2012) 2333-2341.

[14] J. Gupta, C. Nunes, S. Vyas, S. Jonnalagadda, Prediction of solubility parameters and miscibility of pharmaceutical compounds by molecular dynamics simulations, J. Phys. Chem. B 115 (9) (2011) 2014-2023.

[15] M. Belmares, M. Blanco, W.A. Goddard, R.B. Ross, G. Caldwell, S.H. Chou, J. Pham, P.M. Olofson, C. Thomas, Hildebrand and Hansen solubility parameters from molecular dynamics with applications to electronic nose polymer sensors, J. Comput. Chem. 25 (15) (2004) 1814-1826.

[16] N. Bhatnagar, G. Kamath, I. Chelst, J.J. Potoff, Direct calculation of 1-octanol-water partition coefficients from adaptive biasing force molecular dynamics simulations, J. Chem. Phys. 137 (1) (2012) 014502.

[17] T. Ingram, S. Storm, L. Kloss, T. Mehling, S. Jakobtorweihen, I. Smirnova, Prediction of micelle/water and liposome/water partition coefficients based on molecular dynamics simulations, COSMO-RS, and COSMOmic, Langmuir 29 (11) (2013) 3527-3537.

[18] G.E. Karlsson, T.S. Johansson, U.W. Gedde, M.S. Hedenqvist, Determining limonene diffusion in molten polyethylene from within $0.1 \mu$ s molecular dynamics trajectories, Macromolecules 35 (19) (2002) 7453-7459.

[19] M. Tsige, G.S. Grest, Interdiffusion of solvent into glassy polymer films: a molecular dynamics study, J. Chem. Phys. 121 (15) (2004) 7513-7519.

[20] S. Neyertz, D. Brown, Molecular dynamics study of carbon dioxide sorption and plasticization at the interface of a glassy polymer membrane, Macromolecules 46 (6) (2013) 2433-2449.

[21] J.H. Hildebrand, R.L. Scott, The Solubility of Nonelectrolytes, 3rd ed., Reinhold, New York, 1983.

[22] B.C. Hancock, P. York, R.C. Rowe, The use of solubility parameters in pharmaceutical dosage form design, Int. J. Pharm. 148 (1) (1997) 1-21.

[23] Y. Sugita, Y. Okamoto, Replica-exchange molecular dynamics method for protein folding, Chem. Phys. Lett. 314 (1-2) (1999) 141-151.

[24] A. Laio, M. Parrinello, Escaping free-energy minima, Proc. Natl. Acad. Sci. U. S A. 99 (20) (2002) 12562-12566.

[25] B. Isralewitz, M. Gao, K. Schulten, Steered molecular dynamics and mechanica functions of proteins, Curr. Opin. Struct. Biol. 11 (2) (2001) 224-230.

[26] D. Hamelberg, J. Mongan, J.A. McCammon, Accelerated molecular dynamics: a promising and efficient simulation method for biomolecules, J. Chem. Phys. 120 (24) (2004) 11919-11929.

[27] J. Kang, C. Wang, D. Li, G. He, H. Tan, Nanoscale crosslinking in thermoset polymers: a molecular dynamics study, Phys. Chem. Chem. Phys. 17 (25) (2015) 16519-16524.

[28] C. Jang, T.W. Sirk, J.W. Andzelm, C.F. Abrams, Comparison of crosslinking algorithms in molecular dynamics simulation of thermosetting polymers, Macromol. Theory Simul. 24 (3) (2015) 260-270.

[29] M. Bonomi, D. Branduardi, G. Bussi, C. Camilloni, D. Provasi, P. Raiteri, D Donadio, F. Marinelli, F. Pietrucci, R.A. Broglia, M. Parrinello, PLUMED: a portable plugin for free-energy calculations with molecular dynamics, Comput. Phys. Commun. 180 (10) (2009) 1961-1972.

[30] W.L. Jorgensen, D.S. Maxwell, J. Tirado-Rives, Development and testing of the OPLS all-atom force field on conformational energetics and properties of organic liquids, J. Am. Chem. Soc. 118 (45) (1996) 11225-11236.

[31] W.L. Jorgensen, J. Chandrasekhar, J.D. Madura, R.W. Impey, M.L. Klein, Comparison of simple potential functions for simulating liquid water, J. Chem. Phys. 79 (2) (1983) 926-935.

[32] J.-P. Ryckaert, G. Ciccotti, H.J.C. Berendsen, Numerical integration of the cartesian equations of motion of a system with constraints: molecular dynamics of n-alkanes, J. Comput. Phys. 23 (3) (1977) 327-341.

[33] R.W. Hockney, J.W. Eastwood, Computer Simulation using Particles, CRC Press, 1988

[34] S. Plimpton, Fast parallel algorithms for short-range molecular dynamics, J. Comput. Phys. 117 (1) (1995) 1-19. 